\title{
No association between static and dynamic postural control and $A C L$ injury risk among female elite handball and football players: a prospective study of 838 players
}

\author{
Kathrin Steffen, ${ }^{1}$ Agnethe Nilstad, ${ }^{1}$ Tron Krosshaug, ${ }^{1}$ Kati Pasanen, ${ }^{2}$ \\ Aleksander Killingmo, ${ }^{1}$ Roald Bahr ${ }^{1}$
}

${ }^{1}$ Department of Sports

Medicine, Oslo Sports Trauma

Research Center, Norwegian

School of Sport Sciences, Oslo,

Norway

${ }^{2}$ Tampere Research Center of Sports Medicine, UKK Institute for Health Promotion Research, Tampere, Finland

\section{Correspondence to}

Dr Kathrin Steffen, Department of Sports Medicine, Oslo Sports Trauma Research Center, Norwegian School of Sport Sciences, PB 4014 Ullevaal Stadion, Oslo N-0806, Norway; kathrin.steffen@nih.no

Accepted 28 December 2016

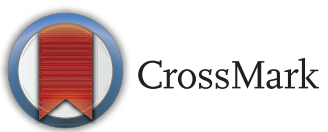

To cite: Steffen $\mathrm{K}$ Nilstad A, Krosshaug T, et al. Br I Sports Med 2017:51:253-259.

\section{ABSTRACT}

Background Research on balance measures as potential risk factors for $\mathrm{ACL}$ injury is limited.

Objective To assess whether postural control was associated with an increased risk for $\mathrm{ACL}$ injuries in female elite handball and football players.

Method Premier league players were tested in the preseason and followed prospectively for $\mathrm{ACL}$ injury risk from 2007 through 2015. At baseline, we recorded player demographics, playing experience, $\mathrm{ACL}$ and ankle injury history. We measured centre of pressure velocity in single-leg stabilisation tests and reach distances in the Star Excursion Balance Test. To examine the stability of postural control measures over time, we examined their short-term and long-term reproducibility. We generated logistic regression models, 1 for each of the proposed risk factors.

Results A total of $55(6.6 \%)$ out of 838 players (age $21 \pm 4$ years; height $170 \pm 6 \mathrm{~cm}$; body mass $66 \pm 8 \mathrm{~kg}$ ) sustained a non-contact $\mathrm{ACL}$ injury after baseline testing (1.8 \pm 1.8 years). When comparing normalised balance measures between injured and uninjured players in univariate analyses, none of the variables were statistically associated with $\mathrm{ACL}$ injury risk. Short-term and long-term reproducibility of the selected variables was poor. Players with a previous $A C L$ injury had a 3 -fold higher risk of sustaining a new ACL injury compared with previously uninjured players (OR 2.9, Cl 1.4 to 5.7$)$.

Conclusions None of postural control measures examined were associated with increased $A C L$ injury risk among female elite handball and football players. Hence, as measured in the current investigation, the variables included cannot be used to predict $A C L$ injury risk.

\section{INTRODUCTION}

Although the aetiology of ACL injuries is not fully understood, they are likely multifactorial in nature, thought to be related to a combination of neuromuscular, biomechanical, anatomical, hormonal and genetic factors. ${ }^{12}$ Typically, ACL injuries occur among females in pivoting sports, characterised by rapid changes of direction and frequent single-leg landings, often with the athlete out of balance and almost always without direct contact to the knee, as, for example, in handball and football. ${ }^{3-6}$ Poor postural control has been suggested to play a crucial role in injury causation ${ }^{6-8}$ although the association between knee kinematics and future ACL injury risk seems to be weak. ${ }^{9}$
The role of balance is believed to be a critical component of neuromuscular control ${ }^{10}$ and as a modifiable risk factor contributes to limit mediolateral knee displacement and loading during dynamic activities. ${ }^{11} 12$ Balance exercises seem to represent a key component of effective ACL injury prevention programmes, which tend to focus on frontal plane knee control during static and dynamic tasks. ${ }^{13-16}$ Balance training using a wobble board has been shown effective in improving static and dynamic balance and reducing sports-related injuries among healthy adolescents. ${ }^{17-19}$ Also, clinicians often use postural control to evaluate deficits resulting from injury and the progress during rehabilitation protocols. $^{20}$

The Star Excursion Balance Test (SEBT) challenges lower limb strength and range of motion and is widely used as a clinical assessment tool for dynamic postural control. ${ }^{21} 22$ Reduced performance in the SEBT, displayed as lower reach distances, has also been linked to an increased likelihood of lower limb injuries. ${ }^{23-25}$

In a small prospective cohort study on 278 NCAA division 1 college athletes (9 ACL injuries), baseline time to stabilisation for backward, forward, medial and lateral single-leg jump-landing tasks were assessed, and the OR for an ACL injury increased threefold for every second these athletes took longer to stabilise following backward jump landing, indicating a significant, albeit weak, association between poor postural control and ACL injury risk. ${ }^{26}$

To date, little research exists quantifying balance measures as potential risk factors for ACL injury. Thus, the purpose of this prospective cohort study was to assess whether static and dynamic postural control were associated with an increased risk for ACL injuries in female elite handball and football players.

\section{METHODS}

\section{Study design and participants}

This investigation represents secondary analyses of data from a cohort study designed to examine risk factors for non-contact ACL injuries in female elite handball and football players. ${ }^{27}$ Data were collected over an 8-year period (2007-2015). Players with a first-team contract who were expected to play in the premier league during the 2007 season were eligible for participation. From 2008 through 2014, new teams advancing to the premier league 
and new players from included teams were invited for preseason tests. From 2009, we also included football players from the female premier league. In total, we have baseline screening data of 429 handball and 451 football players, of which 838 players were included in the current paper (figure 1).

We recorded all complete ACL injuries from the start of screening tests in 2007, through May 2015. For any ACL injury occurring during regular team training or competition, we contacted the injured player by phone to obtain detailed medical data and a description of the injury situation. The injury mechanisms were self-reported as contact (ie, direct contact to the lower extremity), indirect contact (ie, contact with other body parts) or non-contact. Injuries were categorised into two groups, non-contact/indirect contact or contact. ${ }^{6}$ All ACL injuries were verified by MRI and/or arthroscopy.

\section{Risk factor screening tests}

The balance tests included in the present study were part of a comprehensive test battery to assess potential demographic, neuromuscular, two-dimensional (2D) and 3D biomechanical, anatomical, and genetic risk factors for an ACL injury. For all players, the screening tests were conducted at the Norwegian School of Sport Sciences in the preseason: June through August for handball and February through March for football. Each player spent about 7 hours in total to complete the screening, which also included information, warm-up trials, as well as a lunch break. We asked all players to complete a questionnaire to collect data on demographics, elite playing experience, histories of any previous injuries to the ACL or ankle injuries 1 year prior to testing. To examine the short-term and long-term reproducibility of the selected balance tests, we also assessed two groups of athletes twice.

\section{Single-leg stabilisation}

We quantified balance based on centre of pressure (COP) measures on a balance platform (Good Balance system, Metitur, Jyväskylä, Finland). The Good Balance force platform system is an equilateral triangle $(800 \mathrm{~mm})$ that is connected to a threechannel DC amplifier with an A/D converter and uses a sampling frequency of $200 \mathrm{~Hz}$. During two types of single-leg balance tests, we measured the mean velocity of COP in mediolateral (ML) and anterior-posterior (AP) directions $(\mathrm{mm} / \mathrm{s})$, as well as the area of the $95 \%$ confidence ellipse $\left(\mathrm{mm}^{2}\right)$. To control for the possible influence of the higher COP excursions among taller players, we adjusted the results for objectively measured player height $(\mathrm{cm}) .^{28}$

Starting on the preferred kicking leg, we asked the players to maintain balance for $20 \mathrm{~s}$ with arms resting in front of the body while standing on an unstable surface (Airex foams, $40 \mathrm{~cm} \times 50$ $\mathrm{cm}, 7 \mathrm{~cm}$ thick; Alusuisse Airex, Sins, Switzerland) in (1) a purely static position on one foam pad, and (2) following a drop down from $30 \mathrm{~cm}$ height, stabilising on two foams on top of each other. The foam pads had an antifriction layer underneath to prevent sliding on the force plate or between the pads. The test order was the same for all players. To assess landing stability, the drop down test was added to the screening battery in 2009. All players were allowed one practice trial. For both types of tests, the trial was discarded and repeated if the player (1) failed to maintain unilateral stance by moving the stance foot from the initial position, (2) removed the resting arms from the front of her body or (3) got support from the contralateral leg by touching the testing leg. The mean value of two trials for each leg was kept for analyses.

We used the simplified SEBT ${ }^{26}$ to assess dynamic stability and postural control, combined with lower limb strength and range of motion. From a centre point, three tape measures were attached to the floor in the anteromedial, medial and posteromedial directions. The medial direction was oriented perpendicular to the foot placed on the tape measure, and relative to this tape measure, the anteromedial and posteromedial directions were at a $45^{\circ}$ angle.

While maintaining a single-legged stance on the tape measure, the grid midpoint, we asked the player with hands on her waist to reach as far as possible with her contralateral leg in all three directions, starting anteriorly and moving posteriorly in three separate trials. There were no instructions given on lower limb control while balancing; however, hands had to be held at the waist during the testing. Starting with balancing on the preferred kicking leg, we measured the maximal reach distance (to the closest $\mathrm{cm}$ ) to the point where the most distal part of the contralateral foot reached. All players were allowed one practice trial in each direction. The trial was discarded and repeated if the player (1) failed to maintain unilateral stance by lifting or moving the stance foot from the grid, (2) removed her hands

\section{Figure 1}

880 unique player-tests eligible for analyses

\section{9 handball players}

Excluded players

Missing balance data, non-contact $A C L$ injury $(\mathrm{N}=3)$

Missing balance data $(\mathrm{N}=11)$

Contact $A C L$ injury $(\mathrm{N}=6)$
451 football players

Excluded players

Missing balance data $(\mathrm{N}=19)$

Contact $\mathrm{ACL}$ injury $(\mathrm{N}=3)$

Final analyses

838 players; 55 ACL injured legs, 1671 ACL uninjured legs

409 handball players

$25 \mathrm{ACL}$ injured legs
429 football players

$30 \mathrm{ACL}$ injured legs

$828 \mathrm{ACL}$ uninjured legs 
from the waist, (3) touched down with the reach foot and thereby failing to return the reach foot to the starting position. The mean out of three trials in each direction, normalised for leg length, was included in the analyses. Leg length was measured in standing position as the distance from hip joint centre to malleolus as part of $3 \mathrm{D}$ motion analysis. ${ }^{27}$

\section{Ethics approval}

The Regional Committee for Medical Research Ethics, South-Eastern Norway Regional Health Authority and the Norwegian Social Science Data Services approved the study. Players signed a written informed consent form before inclusion, including parental consent for players aged $<18$ years.

\section{Statistical protocol}

Data were analysed using STATA, V.12 (StataCorp, College station, Texas, USA), and descriptive data are presented as means with SDs and frequencies with corresponding percentages. Balance measures are presented as absolute and normalised values. For players sustaining more than one ACL injury following baseline testing, we only included their first noncontact injury as the main outcome in the analyses.

Demographic data and baseline screening results were compared between players with and without a new ACL injury using $\chi^{2}$ tests for categorical data, Student's t-test for continuous variables when the criterion of independency was fulfilled, or by using robust regression models to account for dependencies between legs. We calculated ORs with 95\% CIs for players with and without ACL and ankle injury history. For the final analyses, the significance level was set at $\mathrm{p}<0.05$.

We selected our candidate variables according to hypotheses taken from the literature available until 2006, ${ }^{17} 2931$ and followed a protocol with predefined procedures: following the univariate analyses, we intended to investigate all candidate risk factors with a $\mathrm{p}$ value of $<0.20$ further in a multivariate regression model to explore the association between candidate risk factors and ACL injury, and to adjust for differences in: (1) sport, (2) ACL injury history and (3) ankle injury history.

To examine the stability of balance/postural control measures over time, we retested 144 players (aged 20.9 \pm 3.2 years) $1-5$ years after the first test session $(2.2 \pm 0.8$ years). For those players, we included their first test session results into the risk factor analyses. We also examined short-term reproducibility on 42 similar age elite-level ball sport athletes and sport and exercise students; 26 of these completed the retest session within 3-10 days, while 16 completed the retest session 67 weeks after the first test session. We calculated the mean test difference, the standard method error (SEM) and the minimal detectable change (MDC).

\section{RESULTS}

A total of 838 players were included in the final analyses, 409 handball and 429 football players (figure 1). Player demographics and injury history are presented in table 1.

During follow-up through May 2016, we recorded 80 ACL injuries in 67 players. Of those, 12 players sustained multiple ACL injuries after baseline testing (11 players with 2 injuries and 1 player with 3 injuries). Of the 67 index injuries suffered by these players, we recorded 9 as contact and 58 as noncontact/indirect contact. Three players with a non-contact injury had to be excluded due to missing postural control data, leaving us with 55 non-contact ACL injuries for analyses. The mean time between balance testing and a non-contact ACL injury was $1.8 \pm 1.8$ years.

Players with a new ACL injury following testing did not differ significantly from those who remained free from ACL injury for any of the demographic or training history data. Twelve players with a previous ACL injury $(3.5 \pm 2.5$ years before baseline screening) sustained a new ACL rupture; four of these reruptured the same knee and eight suffered an ACL injury to the contralateral knee.

\section{Univariate risk analysis}

Among the 55 players who went on to suffer a new ACL injury, there was no difference between their injured and uninjured leg for any of the postural sway or dynamic balance measurements ( $p>0.05$; table 2 ). This was also the case when we repeated the analyses after removing all players with a previous ACL injury. The OR of sustaining a new ACL injury among those with a previous ACL injury compared with those with no ACL injury history was 2.86 (95\% CI 1.44 to 5.69 ).

A total of 377 players (45\%) reported at least one ankle injury during the previous year, but there was no difference in ACL injury risk between players with or without a history of ankle injury $(p=0.46)$, including the number of ankle sprains during the year preceding testing $(\mathrm{p}=0.16)$.

When comparing normalised postural sway and balance measures between injured and uninjured legs, none of the selected

Table 1 Demographics, training and injury history of all players, as well as subgroups of players with $(\mathrm{N}=55)$ and without a new/recurrent $\mathrm{ACL}$ injury following testing $(\mathrm{N}=783)$

\begin{tabular}{|c|c|c|c|c|}
\hline & $\begin{array}{l}\text { All players } \\
\mathrm{N}=838\end{array}$ & $\begin{array}{l}\text { Players with new/recurrent } \mathrm{ACL} \\
\mathrm{N}=55\end{array}$ & $\begin{array}{l}\text { Uninjured players } \\
\mathrm{N}=783\end{array}$ & $p$ Value \\
\hline Age (years) & $21.0(4.0)$ & $20.4(3.5)$ & $21.0(4.0)$ & 0.27 \\
\hline Height $(\mathrm{cm})$ & $169.6(6.4)$ & $170.8(7.1)$ & $169.5(6.3)$ & 0.32 \\
\hline Body mass $(\mathrm{kg})$ & $66.3(8.0)$ & $67.6(8.5)$ & $66.2(7.9)$ & 0.20 \\
\hline BMI $\left(\mathrm{kg} / \mathrm{m}^{2}\right)$ & $23.0(2.1)$ & $23.2(2.0)$ & $23.0(2.1)$ & 0.44 \\
\hline Age when starting elite-level play (years) & $18.3(2.8)$ & $17.9(2.8)$ & $18.3(2.8)$ & 0.31 \\
\hline Seasons at elite level (\#) & $2.5(3.3)$ & $2.5(2.9)$ & $2.5(3.3)$ & 0.92 \\
\hline In-season training (hours/week) & $9.8(2.3)$ & $9.4(1.6)$ & $9.8(2.4)$ & 0.18 \\
\hline Off-season training (hours/week) & $8.6(3.5)$ & $8.8(3.2)$ & $8.5(3.5)$ & 0.62 \\
\hline Previous $\mathrm{ACL}$ injury (\#)* & $81(10.0)$ & $12(22.2)$ & $69(8.5)$ & 0.007 \\
\hline Ankle injury previous year $(\#)^{*}$ & $377(45.0)$ & $23(41.8)$ & $354(45.2)$ & 0.46 \\
\hline
\end{tabular}


Table 2 Normalised postural control for injured and contralateral non-injured leg among players with a new/recurrent $A C L$ injury (N=55)

\begin{tabular}{|c|c|c|c|c|}
\hline & $\begin{array}{l}A C L \text { injured legs } \\
N=55\end{array}$ & $\begin{array}{l}\text { Uninjured legs } \\
N=55\end{array}$ & $\begin{array}{l}\Delta \\
95 \% \mathrm{Cl}\end{array}$ & $p$ Value \\
\hline \multicolumn{5}{|c|}{ Balance platform test (static)* } \\
\hline ML speed & $0.12(0.03)$ & $0.12(0.04)$ & $0(-0.008$ to 0.005$)$ & 0.66 \\
\hline AP speed & $0.14(0.04)$ & $0.14(0.04)$ & $0(-0.007$ to 0.004$)$ & 0.67 \\
\hline $95 \%$ centile areal & $4.7(1.6)$ & $4.8(1.9)$ & $-0.1(-0.5$ to 0.4$)$ & 0.81 \\
\hline \multicolumn{5}{|c|}{ Balance platform test (drop down)* } \\
\hline ML speed & $0.25(0.09)$ & $0.25(0.09)$ & $0(-0.050$ to 0.056$)$ & 0.88 \\
\hline AP speed & $0.42(0.20)$ & $0.38(0.09)$ & $0.04(-0.053$ to 0.132$)$ & 0.38 \\
\hline $95 \%$ centile areal & $16.9(8.0)$ & $16.6(7.9)$ & $0.3(-3.6$ to 4.2$)$ & 0.88 \\
\hline \multicolumn{5}{|c|}{ Star Excursion Balance Test* } \\
\hline Anteromedial & $0.84(0.08)$ & $0.83(0.08)$ & $0.01(-0.004$ to 0.021$)$ & 0.18 \\
\hline Medial & $0.86(0.08)$ & $0.86(0.08)$ & $0.01(-0.003$ to 0.021$)$ & 0.14 \\
\hline Posteromedial & $0.94(0.08)$ & $0.93(0.08)$ & $0.01(-0.001$ to 0.024$)$ & 0.06 \\
\hline
\end{tabular}

Table 3 Normalised postural control with mean (SD)

\begin{tabular}{|c|c|c|c|c|c|c|c|c|c|}
\hline & \multicolumn{3}{|l|}{ All players } & \multicolumn{3}{|c|}{ Handball players } & \multicolumn{3}{|c|}{ Football players } \\
\hline & $\begin{array}{l}\mathrm{ACL} \\
\text { injured legs }\end{array}$ & $\begin{array}{l}\mathrm{ACL} \\
\text { uninjured legs }\end{array}$ & p Value & $\begin{array}{l}\mathrm{ACL} \\
\text { injured legs }\end{array}$ & $\begin{array}{l}\mathrm{ACL} \\
\text { uninjured legs }\end{array}$ & p Value & $\begin{array}{l}\mathrm{ACL} \\
\text { injured legs }\end{array}$ & $\begin{array}{l}\mathrm{ACL} \\
\text { uninjured legs }\end{array}$ & p Value \\
\hline Balance platform test (static)* & $\mathrm{N}=44$ & $\mathrm{~N}=1440$ & & $\mathrm{~N}=20$ & $\mathrm{~N}=698$ & & $\mathrm{~N}=24$ & $\mathrm{~N}=742$ & \\
\hline Speed ML ((mm/s)/cm) & $0.12(0.03)$ & $0.12(0.05)$ & 0.76 & $0.13(0.04)$ & $0.13(0.04)$ & 0.92 & $0.11(0.03)$ & $0.12(0.05)$ & 0.63 \\
\hline Speed AP $((\mathrm{mm} / \mathrm{s}) / \mathrm{cm})$ & $0.14(0.03)$ & $0.14(0.04)$ & 0.94 & $0.15(0.04)$ & $0.14(0.04)$ & 0.49 & $0.13(0.02)$ & $0.13(0.05)$ & 0.45 \\
\hline $95 \%$ centile areal $\left(\left(\mathrm{mm}^{2}\right) / \mathrm{cm}\right)$ & $4.6(1.4)$ & $4.8(1.7)$ & 0.38 & $5.0(1.6)$ & $5.0(1.8)$ & 0.93 & $4.3(1.1)$ & $4.6(1.6)$ & 0.20 \\
\hline Balance platform test (drop down)* & $\mathrm{N}=18$ & $\mathrm{~N}=633$ & & $\mathrm{~N}=9$ & $\mathrm{~N}=252$ & & $\mathrm{~N}=9$ & $\mathrm{~N}=381$ & \\
\hline Speed ML $((\mathrm{mm} / \mathrm{s}) / \mathrm{cm})$ & $0.25(0.10)$ & $0.25(0.08)$ & 0.70 & $0.30(0.10)$ & $0.25(0.08)$ & 0.14 & $0.21(0.08)$ & $0.24(0.08)$ & 0.24 \\
\hline Speed AP $((\mathrm{mm} / \mathrm{s}) / \mathrm{cm})$ & $0.43(0.21)$ & $0.39(0.15)$ & 0.48 & $0.45(0.19)$ & $0.39(0.15)$ & 0.34 & $0.41(0.24)$ & $0.39(0.15)$ & 0.88 \\
\hline $95 \%$ centile areal $\left(\left(\mathrm{mm}^{2}\right) / \mathrm{cm}\right)$ & $17.0(8.3)$ & $16.2(10.1)$ & 0.70 & $17.1(7.9)$ & $16.8(13.0)$ & 0.92 & $16.9(9.2)$ & $15.8(7.6)$ & 0.72 \\
\hline Star Excursion Balance Test* & $\mathrm{N}=55$ & $\mathrm{~N}=1517$ & & $\mathrm{~N}=25$ & $\mathrm{~N}=747$ & & $\mathrm{~N}=30$ & $\mathrm{~N}=770$ & \\
\hline Anteromedial $(\mathrm{cm} / \mathrm{cm})$ & $0.83(0.07)$ & $0.84(0.06)$ & 0.38 & $0.86(0.08)$ & $0.86(0.07)$ & 0.81 & $0.81(0.06)$ & $0.83(0.06)$ & 0.11 \\
\hline Medial $(\mathrm{cm} / \mathrm{cm})$ & $0.87(0.08)$ & $0.87(0.07)$ & 0.42 & $0.89(0.08)$ & $0.89(0.07)$ & 0.89 & $0.84(0.07)$ & $0.86(0.06)$ & 0.22 \\
\hline Posteromedial $(\mathrm{cm} / \mathrm{cm})$ & $0.94(0.08)$ & $0.95(0.07)$ & 0.25 & $0.97(0.08)$ & $0.96(0.08)$ & 0.77 & $0.92(0.07)$ & $0.94(0.06)$ & 0.06 \\
\hline
\end{tabular}

variables turned out to be candidate risk factors in univariate risk analyses ( $p>0.20$; table 3$)$. Therefore, we did not conduct multivariate analyses. This was also the case when we repeated the analyses after removing all players with a history of previous ACL injury.

\section{Change of postural control measures over time (reproducibility)}

With an average time of 2.2 (SD 0.8) years between the two test sessions for 144 elite-level players, systematic improvements were observed in postural control measures (1-14\% for postural sway measures, and 3-4\% for functional balance). However, for both postural control measures, the random error was greater than the systematic change, as shown by the SEM and MDC values (table 4). The same was the case for the short-term reproducibility (table 5).

\section{DISCUSSION}

The main findings of this large prospective cohort study to better understand the aetiology of ACL injury do not lend support to postural control as risk factors of importance. Following female elite athletes to ACL injuries as main outcome measure, we could not detect any association between postural control and ACL injury risk. However, it should be noted that the short-term and long-term reproducibility of the variables selected was poor.

\section{Postural sway, dynamic postural balance and injury risk}

Neither postural sway nor dynamic postural control measures in single-leg stabilisation differed between players suffering an ACL injury after the baseline screening and uninjured players. Little research exists examining dynamic, functional balance measures or sway velocity with ACL injury risk as outcome measure. Sway velocity reflects the neuromuscular response following a specific movement task; lower sway velocity suggests a superior response to the balance challenge. ${ }^{31}$ Consequently, we expected players who went on to suffer an ACL injury to display greater sway velocities in the $\mathrm{AP}$ and $\mathrm{ML}$ directions, covering a larger COP area than those who remained free of injury. Similarly, we expected players with a new ACL injury to perform worse on 
Table 4 Long-term (1-5 years) stability of static and dynamic postural control for 144 players (right (R) and left (L) leg)

\begin{tabular}{|c|c|c|c|c|c|c|c|c|}
\hline & \multicolumn{2}{|c|}{ Session 1 baseline } & \multicolumn{3}{|c|}{ Session difference } & \multirow[b]{2}{*}{ SEM } & \multirow[b]{2}{*}{ Per cent } & \multirow[b]{2}{*}{ MDC } \\
\hline & Mean & SD & Mean & Per cent & $95 \% \mathrm{Cl}$ & & & \\
\hline \multicolumn{9}{|l|}{ Balance platform test (static)* } \\
\hline R speed ML (mm/s) & 22.2 & 6.7 & -3.1 & 14.0 & -4.0 to -2.1 & 3.7 & 16.7 & 10.2 \\
\hline R speed AP (mm/s) & 23.3 & 5.3 & -1.1 & 4.7 & -2.0 to -0.3 & 3.3 & 14.2 & 9.1 \\
\hline R $95 \%$ centile areal $\left(\mathrm{mm}^{2}\right)$ & 791.3 & 260.5 & -7.0 & 0.9 & -54.2 to 40.2 & 187.8 & 23.7 & 735.7 \\
\hline L speed ML (mm/s) & 21.7 & 6.6 & -2.7 & 12.4 & -3.6 to -1.8 & 3.7 & 17.1 & 10.2 \\
\hline L speed AP (mm/s) & 23.2 & 5.6 & -1.7 & 7.3 & -2.4 to -0.9 & 0.6 & 2.6 & 1.7 \\
\hline L $95 \%$ centile areal $\left(\mathrm{mm}^{2}\right)$ & 790.2 & 249.6 & -44.7 & 5.6 & -88.5 to -0.9 & 173.9 & 22.0 & 482.0 \\
\hline \multicolumn{9}{|l|}{ Star Excursion Balance Test } \\
\hline $\mathrm{R}$ anteromedial $(\mathrm{cm})$ & 74.1 & 5.8 & 2.6 & 3.5 & 1.9 to 3.4 & 3.2 & 4.3 & 8.9 \\
\hline R medial $(\mathrm{cm})$ & 77.0 & 6.0 & 3.3 & 4.3 & 2.7 to 4.0 & 2.8 & 3.6 & 7.8 \\
\hline R posteromedial $(\mathrm{cm})$ & 84.0 & 6.2 & 3.5 & 4.2 & 2.7 to 4.2 & 3.0 & 3.6 & 8.3 \\
\hline $\mathrm{L}$ anteromedial $(\mathrm{cm})$ & 74.2 & 5.4 & 2.8 & 3.8 & 2.1 to 3.6 & 3.0 & 4.0 & 8.3 \\
\hline L medial $(\mathrm{cm})$ & 77.4 & 6.1 & 2.9 & 3.7 & 2.1 to 3.8 & 3.6 & 4.7 & 10.0 \\
\hline L posteromedial $(\mathrm{cm})$ & 84.7 & 6.1 & 2.6 & 3.1 & 1.6 to 3.5 & 3.8 & 4.5 & 10.5 \\
\hline
\end{tabular}

Data are presented as the session 1 baseline value (mean and SD), the mean session difference (with $95 \% \mathrm{Cl}$ ), the SEM and the MDC. For the Good Balance test negative values denote an improvement from session 1 to 2, while for the Star Excursion Balance Test positive values represent an improved test score.

$\mathrm{AP}$, anterior-posterior; MDC, minimal detectable change; ML, mediolateral; SEM, SE of measurement.

Table 5 Short-term (1-7 weeks) stability of static and dynamic postural control for 144 players (right (R) and left (L) leg)

\begin{tabular}{|c|c|c|c|c|c|c|c|c|}
\hline & \multicolumn{2}{|c|}{ Session 1 baseline } & \multicolumn{3}{|c|}{ Session difference } & \multirow[b]{2}{*}{ SEM } & \multirow[b]{2}{*}{ Per cent } & \multirow[b]{2}{*}{ MDC } \\
\hline & Mean & SD & Mean & Per cent & $95 \% \mathrm{Cl}$ & & & \\
\hline \multicolumn{9}{|l|}{ Balance platform test (static)* } \\
\hline R speed $M L(m m / s)$ & 20.1 & 6.0 & -1.7 & 8.5 & -3.7 to 0.4 & 3.9 & 19.4 & 10.8 \\
\hline R speed AP $(\mathrm{mm} / \mathrm{s})$ & 21.9 & 5.9 & -3.2 & 14.6 & -5.2 to -1.2 & 3.8 & 17.4 & 10.5 \\
\hline R $95 \%$ centile areal $\left(\mathrm{mm}^{2}\right)$ & 886.4 & 268.6 & -93.8 & 10.6 & -204.0 to 16.3 & 208.6 & 23.5 & 577.8 \\
\hline L speed ML $(\mathrm{mm} / \mathrm{s})$ & 18.7 & 5.7 & -1.4 & 7.5 & -3.7 to 1.0 & 4.4 & 23.5 & 12.2 \\
\hline L speed AP $(\mathrm{mm} / \mathrm{s})$ & 21.0 & 6.4 & -2.2 & 10.5 & -4.6 to 0.3 & 4.7 & 22.4 & 13.0 \\
\hline L $95 \%$ centile areal $\left(\mathrm{mm}^{2}\right)$ & 822.6 & 231.9 & -89.8 & 10.9 & -182.6 to 2.9 & 175.6 & 21.3 & 486.4 \\
\hline \multicolumn{9}{|l|}{ Star Excursion Balance Test } \\
\hline $\mathrm{R}$ anteromedial $(\mathrm{cm})$ & 77.7 & 6.2 & 2.3 & 3.0 & 2.3 (1.3 to 3.3 ) & 2.3 & 3.0 & 6.4 \\
\hline $\mathrm{R}$ medial $(\mathrm{cm})$ & 80.5 & 5.6 & 2.3 & 2.9 & 2.3 (1.4 to 3.3$)$ & 3.3 & 4.1 & 6.1 \\
\hline R posteromedial $(\mathrm{cm})$ & 86.7 & 5.2 & 1.8 & 2.1 & 1.8 (0.6 to 2.9$)$ & 3.6 & 4.2 & 7.2 \\
\hline $\mathrm{L}$ anteromedial $(\mathrm{cm})$ & 78.1 & 6.2 & 1.7 & 2.2 & 1.7 (0.5 to 2.9 ) & 2.8 & 3.6 & 7.8 \\
\hline L medial $(\mathrm{cm})$ & 81.7 & 5.3 & 1.4 & 1.7 & $1.4(0.2$ to 2.6$)$ & 2.8 & 3.4 & 7.8 \\
\hline L posteromedial $(\mathrm{cm})$ & 87.1 & 5.0 & 2.2 & 2.5 & 2.2 (1.1 to 3.4$)$ & 2.7 & 3.1 & 7.5 \\
\hline
\end{tabular}

Data are presented as the session 1 baseline value (mean and SD), the mean session difference (with $95 \% \mathrm{Cl}$ ), the SEM and the MDC. For the Good Balance test negative values denote an improvement from session 1 to session 2, while for the Star Excursion Balance Test positive values represent an improved test score.

$\mathrm{AP}$, anterior-posterior; MDC, minimal detectable change; ML, mediolateral; SEM, SE of measurement.

the SEBT, giving themselves a more unstable stance to reach out far with the contralateral leg.

Thus, our findings seem to be in contrast to those of a recent prospective cohort study with nine ACL injuries, where DuPrey et $a l^{26}$ measured time to stabilisation for a variety of jump-landing tasks in a group of 278 NCAA division I college athletes. They reported threefold increased odds for ACL injury risk with longer stabilisation time, albeit following backward jumps only. For comparisons between these two studies, longer time to stabilisation likely corresponds to higher average sway velocities.

One potential explanation for the apparent discrepancy between studies could be the nature of the test tasks used, postural sway in single-leg stability and dynamic balance in SEBT. The stabilisation challenges to postural control chosen here may simply be inadequate and not be representative for typical handball and football injury situations to produce changes associated with increased ACL injury risk. By asking our players to drop down from a $30 \mathrm{~cm}$ high box before stabilising on two foam pads, we increased the challenge considerably, and both the speed and excursion of the COP increased significantly compared with the purely static task. However, even this more dynamic and challenging task did not discriminate between injured and uninjured players.

We also measured dynamic functional balance with the simplified version of the SEBT, using three test directions slightly different from what is commonly used in the literature. ${ }^{29}$ With the exclusion of the posterolateral test arm, where the reaching leg crosses behind the player, the simplified version of the SEBT may be less challenging. Still, we do not believe that this difference in test procedures is less likely to detect an association with the outcome measure, ACL injury risk. 
In both sports, players jump, land and change direction at high speed while focusing on teammates and opponents. Hence, more sport-specific cutting and jump-landing tasks in combination with single-leg stabilisation could have increased the validity of our test, ${ }^{32}$ however, also lessening the standardisation of the test procedures.

\section{Injury history and injury risk}

The consistent identification of previous injury as a risk factor for a subsequent new injury highlights the importance of avoiding the first injury. In the current study, the odds for sustaining a new ACL injury in the group of players with an ACL injury history were tripled, which is in line with other studies on different athlete groups. ${ }^{33-35}$ Since the injured group was also highly biased by ACL injury (12 of 55 players), we repeated all analyses excluding players with previous injury. However, the results remained the same. Postural stability measurements during the single-leg stance may be a useful predictor of increased risk of non-contact lower extremity injury. ${ }^{36}$ Surprisingly, we could not identify any association between a history of ankle injury the preceding year and ACL injury risk.

\section{Methodological considerations}

When interpreting the findings of the present study, there are several strengths and limitations that should be kept in mind. With almost 900 female elite athletes tested, this is among the largest prospective studies assessing risk factors for ACL injury. Nevertheless, with our homogeneous sample of elite-level athletes, the generalisability to other populations, for example, younger or less fit athletes, is unknown.

Also, even with 55 non-contact ACL injuries included, the study is not sufficiently powered to address more than five candidate risk factors, including covariates, at a time. ${ }^{37}$ As can be seen from simple comparisons between injured versus uninjured legs, and from short-term and long-term reproducibility data, it is clear that none of the factors examined have strong associations with injury risk. In other words, increasing sample size further is unlikely to reveal clinically significant factors.

As used in the present study, the most common and reproducible method for quantifying standing balance is based on COP measures. ${ }^{38-40}$ COP sway velocity is seen as the most reliable measure. ${ }^{38}$ However, test reproducibility in our cohort was poor.

As the players rotated between test stations, the individual start of test station was random, and fatigue during testing could have affected player performance, also during balance testing. Still, the potential effect on fatigue will have been random and balanced out among the, in total, 838 players in the analyses, and between those who went on to injury or did not injure their ACL.

As with all prospective cohort studies, risk factors may have changed after inclusion. The time between baseline balance testing and the main outcome measure, ACL injury, was on average 1.8 years (range 1-89 months). We do not have follow-up information on player exposure to elite-level play, injury history other than new ACL injuries, injury prevention training or other neuromuscular training habits. These are among factors that could influence postural control characteristics, causing misclassification and thus reducing our ability to detect associations with ACL injury risk. To reduce variability for potential changes in risk factors in the cohort, we recalculated our analyses for a subgroup of 23 players with an ACL injury within 1 year following screening. The results on association between balance variables and ACL injury risk did not change.
Also, short-term and long-term test-retest data on our postural control measures showed significant improvements despite large individual variations, implying a learning effect, as late as after 2 years as after a few weeks following the first test session. Large MDC values lesson our ability to detect injury risk factors.

Finally, we relied on interviews with the athlete and medical staff to classify injuries as contact, indirect contact or noncontact. Separate regression analyses with all 67 prospective contact and non-contact ACL injuries included, revealed no changes in either postural control, knee motion control $^{27}$ or peak strength outcomes measures, ${ }^{41}$ documenting that potential misclassification of the mechanism of injury is not likely to change the results of this study.

\section{IMPLICATIONS}

Several meta-analyses on the effect of multicomponent exercise prevention programmes highlight the role of varying neuromuscular training and balance components to be of importance for effective ACL injury risk reduction. ${ }^{42}{ }^{43}$ However, we found no significant difference in either sway velocity, excursion or dynamic balance between injured and uninjured female elite athletes. The selected single-leg stabilisation tests may not have been challenging enough to identify players at risk.

Injury risk among highly compliant female elite handball players was effectively reduced when following a one-season ACL injury prevention programme that almost solely focused on the cut and landing technique, and balance training with knee control. ${ }^{15}$ In a separate intervention study using the same exercise protocol, elite football and handball players increased muscle activation of the medial hamstring muscles prior to landing. ${ }^{44}$ In other words, there may be other benefits of neuromuscular training than simply improving postural control.

In the present prospective cohort of Norwegian female handball and football players, neither isolated motion patterns during drop jump landings ${ }^{27}$ nor lower extremity strength ${ }^{41}$ seem to play a role in ACL injury causation. Hence, combining these neuromuscular variable clusters to address the truly multifactorial nature of ACL injures will not help us in finding associations between those variables and ACL injury risk in our cohort.

Nevertheless, as we still do not understand the mechanisms underpinning effective exercise ACL injury prevention programmes, we highly recommend their continued use, irrespective of player level. ${ }^{13-16}$

\section{CONCLUSION}

None of the postural control measures examined were associated with an increased ACL injury risk among female elite ball

\section{What are the findings?}

- Little research exists quantifying balance measures as potential risk factors for $A C L$ injury.

- We found no significant difference in either sway velocity, excursion or dynamic balance between injured and uninjured female elite athletes.

- Postural control measures, as measured in the current investigation, cannot be used to predict $\mathrm{ACL}$ injury risk.

- Short-term and long-term reproducibility of the variables selected was poor. 


\section{How might it impact on clinical practice in the future?}

- Any of the measured variables studied have shown utility in injury prediction of lower limb injury risk. What has been shown in some studies is a statistically significant association with injury risk, which should not be confused with prediction.

- The selected single-leg stabilisation tests in the current investigation may not have been challenging enough to identify players at risk.

- More sport-specific cutting and jump-landing tasks in combination with single-leg stabilisation will likely increase the validity of testing, however, also reduce the standardisation of test procedures.

- We still do not understand the mechanisms underpinning effective exercise of $\mathrm{ACL}$ injury prevention programmes. Still, we highly recommend their continued use, irrespective of player level.

sport athletes. Hence, as measured in the current investigation, the variables included cannot be used to predict ACL injury risk.

\section{Twitter Follow Agnethe Nilstad @agnethenilstad}

Acknowledgements The authors acknowledge all players in the Norwegian female premier football league and handball league for their participation in this study. The authors thank Helene Vileid, Joar Harøy, and Dr Kam-Ming Mok for their contribution with short-term and long-term reproducibility data and for valuable discussions, and all members of the project group and research assistants who contributed to the data collection. The Oslo Sports Trauma Research Center has been established at the Norwegian School of Sport Sciences through generous grants from the Royal Norwegian Ministry of Culture, the South-Eastern Norway Regional Health Authority, the International Olympic Committee, the Norwegian Olympic Committee \& Confederation of Sport, and Norsk Tipping AS. This study also received financial support from the FIFA Medical Assessment and Research Centre.

Contributors KS, AN, TK, KP, AK and RB contributed to the study design and data recoding preparation. All authors were responsible for the data collection and analysis, and KS wrote the first draft. All authors contributed to the final paper. KS, TK and RB are responsible for the overall content as guarantors.

Competing interests None declared.

Ethics approval The Regional Committee for Medical Research Ethics, South-Eastern Norway Regional Health Authority and the Norwegian Social Science Data Services approved the study.

Provenance and peer review Not commissioned; externally peer reviewed.

Data sharing statement The present paper contains original material only that has not been previously published.

\section{REFERENCES}

1 Svoboda SJ, Owens BD, Harvey TM, et al. The association between serum biomarkers of collagen turnover and subsequent anterior cruciate ligament rupture. Am J Sports Med 2016;44:1687-93.

2 Shultz SJ, Schmitz RJ, Nguyen AD, et al. ACL research retreat V: an update on $A C L$ injury risk and prevention, March 25-27, 2010, Greensboro, NC. J Athl Train 2010;45:499-508.

3 Koga $\mathrm{H}$, Bahr R, Myklebust $\mathrm{G}$, et al. Estimating anterior tibial translation from model-based image-matching of a noncontact anterior cruciate ligament injury in professional football: a case report. Clin J Sport Med 2011;21:271-4.

4 Koga H, Nakamae A, Shima Y, et al. Mechanisms for noncontact anterior cruciate ligament injuries: knee joint kinematics in 10 injury situations from female team handball and basketball. Am J Sports Med 2010;38:2218-25.

5 Waldén $\mathrm{M}$, Hägglund $\mathrm{M}$, Werner J, et al. The epidemiology of anterior cruciate ligament injury in football (soccer): a review of the literature from a gender-related perspective. Knee Surg Sports Traumatol Arthrosc 2011;19:3-10.

6 Olsen $\mathrm{OE}$, Myklebust G, Engebretsen L, et al. Injury mechanisms for anterior cruciate ligament injuries in team handball: a systematic video analysis. Am I Sports Med 2004;32:1002-12
7 Krosshaug T, Nakamae A, Boden BP, et al. Mechanisms of anterior cruciate ligament injury in basketball: video analysis of 39 cases. Am I Sports Med 2007;35:359-67.

8 Waldén M, Krosshaug T, Bjørneboe J, et al. Three distinct mechanisms predominate in non-contact anterior cruciate ligament injuries in male professional football players: a systematic video analysis of 39 cases. Br I Sports Med 2015:49:1452-60.

9 Leppänen M, Pasanen K, Kujala UM, et al. Stiff landings are associated with increased $\mathrm{ACL}$ injury risk in young female basketball and floorball players. Am J Sports Med 2016: Published online first Sep 16.

10 Cortes N, Porter LD, Ambegaonkar JP, et al. Postural stability does not differ among female sports with high risk of anterior cruciate ligament injury. Med Probl Perform Art 2014:29:216-20.

11 Vrbanić TS, Ravlić-Gulan J, Gulan G, et al. Balance index score as a predictive factor for lower sports results or anterior cruciate ligament knee injuries in Croatian female athletes-preliminary study. Coll Antropol 2007;31:253-8.

12 Paterno MV, Myer GD, Ford KR, et al. Neuromuscular training improves single-limb stability in young female athletes. I Orthop Sports Phys Ther 2004;34:305-16

13 Waldén $\mathrm{M}$, Atroshi I, Magnusson $\mathrm{H}$, et al. Prevention of acute knee injuries in adolescent female football players: cluster randomised controlled trial. BMJ 2012;344:e3042.

14 Mandelbaum BR, Silvers HJ, Watanabe DS, et al. Effectiveness of a neuromuscular and proprioceptive training program in preventing anterior cruciate ligament injuries in female athletes: 2-year follow-up. Am J Sports Med 2005;33:1003-10.

15 Myklebust G, Engebretsen L, Braekken IH, et al. Prevention of anterior cruciate ligament injuries in female team handball players: a prospective intervention study over three seasons. Clin J Sport Med 2003;13:71-8.

16 Olsen $\mathrm{OE}$, Myklebust $\mathrm{G}$, Engebretsen $\mathrm{L}$, et al. Exercises to prevent lower limb injuries in youth sports: cluster randomised controlled trial. BMJ 2005;330:449.

17 Emery CA, Cassidy JD, Klassen TP, et al. Effectiveness of a home-based balance-training program in reducing sports-related injuries among healthy adolescents: a cluster randomized controlled trial. CMAJ 2005;172:749-54.

18 Bahr R, Lian O, Bahr IA. A twofold reduction in the incidence of acute ankle sprains in volleyball after the introduction of an injury prevention program: a prospective cohort study. Scand J Med Sci Sports 1997;7:172-7.

19 Verhagen E, van der Beek A, Twisk J, et al. The effect of a proprioceptive balance board training program for the prevention of ankle sprains: a prospective controlled trial. Am I Sports Med 2004;32:1385-93.

20 Gribble PA, Kelly SE, Refshauge KM, et al. Interrater reliability of the star excursion balance test. J Athl Train 2013;48:621-6.

21 Stiffler MR, Sanfilippo JL, Brooks MA, et al. Star Excursion Balance Test performance varies by sport in healthy division I collegiate athletes. I Orthop Sports Phys Ther 2015;45:772-80.

22 Kinzey SJ, Armstrong CW. The reliability of the star-excursion test in assessing dynamic balance. J Orthop Sports Phys Ther 1998;27:356-60.

23 Plisky PJ, Rauh MJ, Kaminski TW, et al. Star Excursion Balance Test as a predictor of lower extremity injury in high school basketball players. J Orthop Sports Phys Ther 2006;36:911-9.

24 Gribble PA, Terada M, Beard MQ, et al. Prediction of lateral ankle sprains in football players based on clinical tests and body mass index. Am J Sports Med 2016:44:460-7.

25 Butler RJ, Lehr ME, Fink ML, et al. Dynamic balance performance and noncontact lower extremity injury in college football players: an initial study. Sports Health 2013:5:417-22.

26 DuPrey KM, Liu K, Cronholm PF, et al. Baseline time to stabilization identifies anterior cruciate ligament rupture risk in collegiate athletes. Am J Sports Med 2016;44:1487-91.

27 Krosshaug T, Steffen K, Kristianslund EK, et al. Vertical drop jump tests cannot predict $\mathrm{ACL}$ injuries: a prospective cohort study of 710 female elite football (soccer) and handball players. Am I Sports Med 2016;44:874-83.

28 Era P, Schroll M, Ytting H, et al. Postural balance and its sensory-motor correlates in 75-year-old men and women: a cross-national comparative study. J Gerontol A Biol Sci Med Sci 1996;51:M53-63.

29 Hertel J, Braham RA, Hale SA, et al. Simplifying the star excursion balance test: analyses of subjects with and without chronic ankle instability. J Orthop Sports Phys Ther 2006;36:131-7.

30 Gribble PA, Hertel J, Plisky P. Using the Star Excursion Balance Test to assess dynamic postural-control deficits and outcomes in lower extremity injury: a literature and systematic review. J Athl Train 2012;47:339-57.

31 McGuine TA, Greene JJ, Best T, et al. Balance as a predictor of ankle injuries in high school basketball players. Clin I Sport Med 2000;10:239-44.

32 Kristianslund EK, Krosshaug T. Comparison of drop jumps and sport-specific sidestep cutting: implications for anterior cruciate ligament injury risk screening. Am J Sports Med 2013;41:684-8.

33 Paterno MV, Rauh MJ, Schmitt LC, et al. Incidence of second ACL injuries 2 years after primary ACL reconstruction and return to sport. Am I Sports Med 2014;42:1567-73. 


\section{Original article}

34 Paterno MV, Rauh MJ, Schmitt LC, et al. Incidence of contralateral and ipsilateral anterior cruciate ligament $(\mathrm{ACL})$ injury after primary $\mathrm{ACL}$ reconstruction and return to sport. Clin J Sport Med 2012;22:116-21.

35 Faude 0 , Junge $A$, Kindermann $W$, et al. Risk factors for injuries in elite female soccer players. Br J Sports Med 2006;40:785-90.

36 Dingenen B, Malfait B, Nijs S, et al. Postural stability during single-leg stance: a prospective evaluation of non-contact lower extremity injury risk. J Orthop Sports Phys Ther 2016;46:650-7.

37 Bahr R, Holme I. Risk factors for sports injuries-a methodological approach. Br J Sports Med 2003:37:384-92.

38 Lin D, Seol H, Nussbaum MA, et al. Reliability of COP-based postural sway measures and age-related differences. Gait Posture 2008;28:337-42.

39 Gerbino PG, Griffin ED, Zurakowski D. Comparison of standing balance between female collegiate dancers and soccer players. Gait Posture 2007;26:501-7.
40 Karst GM, Venema DM, Roehrs TG, et al. Center of pressure measures during standing tasks in minimally impaired persons with multiple sclerosis. J Neurol Phys Ther 2005;29:170-80.

41 Steffen K, Nilstad A, Kristianslund EK, et al. Association between lower extremity muscle strength and noncontact ACL injuries. Med Sci Sports Exerc 2016;48:2082-9.

42 Donnell-Fink LA, Klara K, Collins JE, et al. Effectiveness of knee injury and anterior cruciate ligament tear prevention programs: a meta-analysis. PLOS ONE 2015;10: e0144063.

43 Taylor JB, Waxman JP, Richter SJ, et al. Evaluation of the effectiveness of anterior cruciate ligament injury prevention programme training components: a systematic review and meta-analysis. Br J Sports Med 2015;49:79-87.

44 Zebis MK, Bencke J, Andersen LL, et al. The effects of neuromuscular training on knee joint motor control during sidecutting in female elite soccer and handball players. Clin J Sport Med 2008;18:329-37. 